\title{
Ginsenoside Rg1 Prevents Chronic Stress- Induced Depression-Like Behaviors and Neuronal Structural Plasticity in Rats
}

\author{
Hongluan $\mathrm{Yu}^{\mathrm{a}}$ Cuiqin Fan ${ }^{\mathrm{b}}$ Lejin Yang ${ }^{\mathrm{a}}$ Shuyan $\mathrm{Yu}^{\mathrm{b}}$ Qiqi Song \\ Peng Wang ${ }^{b}$ Xueqin Mao \\ aDepartment of Psychiatry, Qilu Hospital of Shandong University, Jinan, bepartment of Physiology, \\ Shandong University, School of Medicine, Jinan, China
}

\section{Key Words}

Ginsenoside Rg1 • Chronic unpredictable mild stress • Structural plasticity • MiR-134 • BDNF

\begin{abstract}
Background/Aims: Ginsenoside Rg1 has been demonstrated to exhibit neuroprotective effects in various studies. This study aimed to investigate the neuronal mechanisms underlying the neuroprotective and antidepressant-like effects of ginsenoside Rg1 in a rat model of depression. Methods: Chronic unpredictable mild stress was used to induce depression-like behaviors in rats. Transmission electron microscopy was used to observe neuronal synapses within the basolateral amygdala (BLA). The expression of microRNA (miR)-134 in the BLA was verified by real-time quantitative PCR. Finally, the synaptic plasticity-associated proteins CAMPresponse element binding protein (CREB) and brain-derived neurotrophic factor (BDNF) were detected by immunoblotting. Results: Results showed that chronic stress effectively induced depression-like behaviors in rats, which were associated with significant ultrastructural changes within BLA neurons. Moreover, chronic stress decreased the expression of miR-134 in the BLA, which was accompanied by decreased phosphorylation of CREB and decreased expression of BDNF. Remarkably, chronic administration of ginsenoside Rg1 (40 mg/kg, i.p., 5 weeks) significantly ameliorated the neuronal structural abnormalities and biochemical changes induced by chronic stress, as well as preventing depression-like behaviors in these rats. Conclusion: Results suggested that ginsenoside Rg1 may exhibit neuroprotection and antidepressant-like effects by activating the CREB-BDNF system within the BLA in this rat model of depression. Amelioration of depression-like behaviors by ginsenoside Rg1 appears to involve modulation of the synapse-associated factor miR-134 within the BLA. Therefore, these findings demonstrate some of the neuronal mechanisms associated with depression and the therapeutic potential of ginsenoside $\mathrm{Rg} 1$ for use in the treatment of depression in clinical trials.




\section{Cellular Physiology Cell Physiol Biochem 2018;48:2470-2482

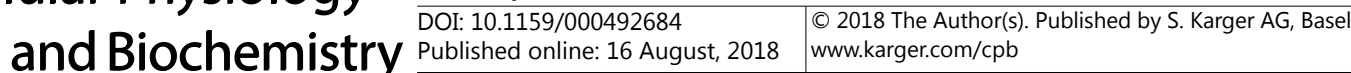 \\ Yu et al.: Ginsenoside Rg1 Prevents Depression Phenotypes}

\section{Introduction}

Depression is a major psychiatric disorder that is related to neuronal structural changes within specific brain regions that result from molecular and cellular changes induced by external stress stimuli [1-3]. Recently, the basolateral amygdala (BLA), which serves as the key locus in mediating initial stages of cortical processing to the amygdala, has been recognized as a critical brain region involved in the pathophysiology of depression $[4,5]$. Neuroimaging studies have shown that chronic or traumatic stress leads to hyperactivity of the amygdala in depressed patients [6-9], and chronic stress increases the number of dendritic spines and dendritic length of principal BLA neurons in animal models of depression [10-12]. Therefore, these studies suggest the possibility that structural and functional plasticity of BLA neurons may contribute to a range of emotionally related behaviors, including depression. However, the molecular mechanisms underlying this neuronal plasticity in dendritic morphology, spine number, or spine distribution within the BLA and how stressors lead to these changes are yet to be determined.

MicroRNAs (miRNAs) have been suggested to be important factors in epigenetic control of neural plasticity in the brain $[13,14]$. MiR-134, a brain-specific miRNA mainly localized to the synapto-dendritic compartment of neurons, has been hypothesized to play a key role in synaptic development and plasticity [15]. Previous studies suggested that miR-134 regulates distinct sets of target genes involved in the formation, maturation, and/ or plasticity of synapses. For example, miR-134 was found to regulate synaptic plasticity and memory formation by inhibiting translation of some plasticity-related proteins such as cAMP response element-binding protein (CREB) and brain derived neurotrophic factor (BDNF) [16]. CREB, a key nuclear transcription factor, has been well documented in neuronal plasticity and long-term memory formation $[17,18]$. Its activation promotes the transcription and translation of BDNF, a crucial regulator involved in providing lifelong protection and regulation of neuronal structure and function [19-21]. It has been demonstrated that chronic stress leads to a reduction in hippocampal BDNF levels in rats [22], and clinical studies also indicated characteristic decreases in BDNF expression in the serum and amygdala of patients with major depressive disorders $[23,24]$. The importance of BDNF has been further supported by findings that showed that local infusion of BDNF into the hippocampus induces antidepressant-like effects in some animal models of depression [25]. However, it is not known whether such effects are involved in chronic stress-induced dysregulation of neuronal plasticity in the BLA and thereby lead to depression-like behaviors.

In recent years, herbal medicines have become a novel and attractive pharmacotherapy in the treatment of depression due to their antidepressant-like effects and high safety margins [26, 27]. Ginsenoside Rg1, the major active ingredient of the herb Panax Ginseng, has been shown to be a potential neuroprotective agent for Alzheimer's disease and other neurological disorders $[28,29]$, and to improve cognitive and memory impairments in animal models [30, 31]. Recent studies have shown that ginsenoside Rg1 exerts antidepressant-like effects by reversing reductions in BDNF protein levels induced by chronic stress in the mouse hippocampus [32]. Such results suggest a possible relationship between the neuroprotective effects and antidepressant-like effects of ginsenoside Rg1. However, the underlying mechanisms of ginsenoside Rg1 in the regulation of neurotrophic factor expression remains unknown. Neurotrophic factor expression is related to structural and functional plasticity of neurons in the pathophysiology of depression, and such understanding of regulation may lead to the discovery of mechanisms underlying the amelioration of depression-like behaviors in these animals.

In this study, we investigated the neuroprotective and antidepressant-like effects of ginsenoside $\operatorname{Rg} 1$ in a chronic unpredictable mild stress (CUMS)-induced animal model of depression. To further explore the possible involvement of neurotrophic activity in mediating the antidepressant-like effects of ginsenoside $\mathrm{Rg} 1$, the role of miR-134-mediated activity of the CREB/BDNF signaling pathway was assessed in the BLA, a specific brain region associated with depression in this animal model. 


\section{Cellular Physiology Cell Physiol Biochem 2018;48:2470-2482 \begin{tabular}{ll|l} 
and Biochemistry Published onlıne: 16 August, 2018 & $\begin{array}{l}\text { (c) } 2018 \text { The Author(s). Published by S. Karger AG, Basel } \\
\text { www.karger.com/cpb }\end{array}$
\end{tabular}}

Yu et al.: Ginsenoside Rg1 Prevents Depression Phenotypes

\section{Materials and Methods}

\section{Animals}

Male Wistar rats weighing 160-180 g were obtained from the Shandong University Animal Centre. All procedures were approved by the Shandong University Animal Care and Use Committee and were performed in accordance with the National Institutes of Health Guide for the Care and Use of Laboratory Animals (National Research Council, 1996). Rats were housed in groups of four per cage in standard plastic cages under controlled temperature $\left(22-24^{\circ} \mathrm{C}\right)$ and humidity $(50 \pm 10 \%)$ conditions with a 12 -h light/dark cycle (lights on at 6:30 a.m.), and were acclimatized to these laboratory conditions for at least 1 week prior to the start of the experiments. Food and water were freely available throughout the experiments except during certain experimental procedures. All efforts were made to minimize pain and the number of animals used in these experiments.

\section{Drugs and treatment}

Ginsenoside Rg1 (Shanghai Winherb Medical S \& T Development Co., Ltd., Shanghai, China) was dissolved in normal saline at a final concentration of $10 \mathrm{mg} / \mathrm{ml}$. The dose and route of ginsenoside $\operatorname{Rg} 1$ administration used in this experiment were chosen based on previous results [33]. In all experiments, drugs were administered intraperitoneally (i.p.) at a constant volume $30 \mathrm{~min}$ prior to stress exposure, which was conducted once a day for 5 weeks. Rats were randomly allocated to one of the following four groups (n = 12/group): (a) non-CUMS, (b) CUMS, (c) ginsenoside Rg1 (40 mg/kg) followed by CUMS, and (d) vehicle (physiological saline, $10 \mathrm{ml} / \mathrm{kg}$ ) followed by CUMS. To habituate rats to the i.p. injections, all rats were administered saline $(10 \mathrm{ml} / \mathrm{kg})$ daily for three days prior to the experiment.

\section{CUMS procedure}

The CUMS procedure was conducted as described previously [34], with minor modifications. Briefly, non-stressed rats were housed in groups of four per cage in standard plastic cages in a room separate from stressed rats to avoid the influence of stress stimuli. Rats in the other three groups were randomly assigned to social isolation accompanied by a weekly stress regime consisting of $24 \mathrm{~h}$ food deprivation followed by 24 $\mathrm{h}$ water deprivation, $45^{\circ}$ cage tilt $(24 \mathrm{~h}), 5 \mathrm{~min}$ cold swimming (at $4^{\circ} \mathrm{C}$ ), $2 \mathrm{~h}$ physical restraint, cage moisture $(24 \mathrm{~h})$, and overnight illumination. One of these stressors was applied individually each day in a random order for a continuous 5 -week period.

\section{Behavioral tests}

Sucrose preference test. Rats were tested for anhedonia using the sucrose preference test as described previously [34], with minor modifications. Briefly, after 5 weeks of CUMS exposure, animals were placed individually in cages to habituate to sucrose solution $(1 \%, \mathrm{w} / \mathrm{v})$ before onset of the sucrose test. During this test, two bottles of $1 \%$ sucrose solution were placed in the cage for the first $24 \mathrm{~h}$, followed by replacement of one of the bottles with a bottle of tap water for the second 24-h period. After the adaptation phase, rats were deprived of food and water for $24 \mathrm{~h}$. Then, rats were permitted free access to the two bottles, one containing $100 \mathrm{ml}$ of sucrose solution (1\%, w/v) and the other $100 \mathrm{ml}$ of tap water, for $3 \mathrm{~h}$. Consumed volumes of sucrose solution and tap water were recorded, and sucrose preference, which is an indicator for assessing anhedonia, was then calculated as sucrose consumption/ (water consumption + sucrose consumption) $\times$ $100 \%$.

Forced swim test. Rats were subjected to the forced swim test as described previously [35, 36]. Briefly, rats were placed individually in a cylinder (height: $80 \mathrm{~cm}$; diameter: $30 \mathrm{~cm}$ ) filled with $50 \mathrm{~cm}$ of water at $25^{\circ} \mathrm{C}$ for two consecutive swim sessions. For the first session (training session), each rat was placed individually in the chamber for 15 min of forced swimming. Twenty-four hours later, the rat was placed in the cylinder for a 5-min session (test session). An observer who was blind to the treatment condition of the animal scored the 5-min test for durations (in seconds) of immobility (floating or only movements required to keep the head above water), swimming, and struggling (climbing walls or diving).

Open field test. The open-field test was performed to measure spontaneous locomotor activity as described previously [37], with minor modifications. In the present study, the test chamber consisted of 


\section{Cellular Physiology Cell Physiol Biochem 2018;48:2470-2482 \begin{tabular}{l|l|l} 
and Biochemistry & DOI: 10.1159/000492684 & $\begin{array}{l}\text { C } 2018 \text { The Author(s). Published by S. Karger AG, Basel } \\
\text { www.karger.com/cpb }\end{array}$
\end{tabular}}

Yu et al.: Ginsenoside Rg1 Prevents Depression Phenotypes

a square plywood arena $(100 \times 100 \times 40 \mathrm{~cm})$ with a black surface covering the inside walls. The floor was divided into 25 equal squares by black lines. The rat was placed in the center of the arena and was allowed to explore freely for $5 \mathrm{~min}$. During the test session, horizontal locomotor activities (segments crossed with all four limbs) and vertical exploratory activities (number of rearings defined as standing on their hind limbs) were counted. The box was cleaned after each test session to remove any olfactory cues.

\section{Quantitative real-time PCR}

Twenty-four hours after behavioral testing, rats were anesthetized with sodium pentobarbital (150 mg/kg, i.p.) and the brain was quickly removed. Then, the BLA was carefully dissected while being maintained on ice, and total RNA was isolated using the RNA rapid extraction kit (Aidlab Biotechnologies, Ltd., Beijing, China) according to the manufacturer's protocol. Two micrograms of total RNA was used to generate cDNA as a template by reverse transcription using the All-in-One ${ }^{\mathrm{TM}}$ miRNA First-Strand cDNA Synthesis Kit (GeneCopoeia, Guangzhou, China). The reverse transcription reaction was amplified using a Bio-Rad CFX96 Detection System (Bio-Rad Laboratories, Hercules, CA). For miRNA amplification, rnomiR-134 (RmiRQP0168, GeneCopoeia) were used as primers and U6 snRNA (RmiRQP9003, GeneCopoeia) was used for miRNA control. Quantitative real-time PCR was performed using the Bio-Rad IQ5 Real Time PCR System (Bio-Rad Laboratories), and the reaction conditions were $95^{\circ} \mathrm{C}$ for $10 \mathrm{~min}$, followed by 40 cycles of $95^{\circ} \mathrm{C}$ for $10 \mathrm{~s}, 55^{\circ} \mathrm{C}$ for $20 \mathrm{~s}$, and $72^{\circ} \mathrm{C}$ for $15 \mathrm{~s}$. The relative fold change in expression of miR-134 was determined by the $\Delta \Delta \mathrm{CT}$ method using Bio-Rad IQ5 Software (Bio-Rad Laboratories).

\section{Electron microscopy analysis}

After the brains were removed, they were processed for transmission electron microscopy analysis after behavioral tests. The BLA $(1 \times 1 \times 1 \mathrm{~mm})$ was carefully dissected while kept on ice and was then placed in $2.5 \%$ glutaraldehyde at $4^{\circ} \mathrm{C}$ for $4 \mathrm{~h}$ after being rinsed in $0.1 \mathrm{M}$ sodium citrate buffer $(\mathrm{pH} 7.4)$. The tissue was then fixed with $1 \%$ osmium tetroxide for $1 \mathrm{~h}$ and subjected to a graded ethanol dehydration series. The tissue was then infiltrated with a mixture of one-half propylene oxide overnight and embedded in resin. Ultrathin sections (70 nm thick) were cut using an ultramicrotome (Ultracut N, Leica, Wetzlar, Germany) and then stained with $4 \%$ uranyl acetate for $20 \mathrm{~min}$ followed by $0.5 \%$ lead citrate for $5 \mathrm{~min}$. The ultrastructure of BLA neurons was observed under a transmission electron microscope (Philips Tecnai 20 U-Twin, Holland). Analysis of synaptic density was carried out by the size-frequency method as described previously [38, 39]. In this study, at least 30 micrographs were randomly taken from each rat for analysis. The measurement was performed by researchers who were blind to the treatment condition of the animal, and was assisted by the use of Image J analysis software (National Institutes of Health, Bethesda, MD).

\section{Western blot analysis}

In this study, the BLA of each rat was analyzed separately. The BLA was immediately homogenized in $600 \mu \mathrm{l}$ ice-cold lysis buffer with protease inhibitor cocktail for a 30-min incubation period. The homogenate was centrifuged at $14,000 \mathrm{rpm}$ for $10 \mathrm{~min}$ at $4^{\circ} \mathrm{C}$ and supernatants collected for protein concentration measurement. Thirty micrograms of proteins were then loaded onto each lane and electrophoretically separated on $12 \%$ sodium dodecyl sulfate polyacrylamide gel electrophoresis gels and transferred for western blot analysis. Primary antibodies used were polyclonal rabbit anti-BDNF (1:500, Santa Cruz Biotechnology, Santa Cruz, CA), anti-CREB (1:1000, Cell Signaling Technology, Danvers, MA), and anti-

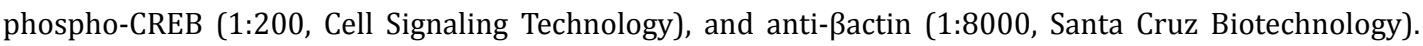
Horseradish peroxidase-conjugated antibody (1:5000, Santa Cruz Biotechnology) was used as the secondary antibody. A high-sensitivity enhanced chemiluminescence kit (GE Healthcare, Little Chalfont, UK) was used for analysis. Protein band densities, which were combined for each group (six rats per group), were quantified using Image J software (National Institutes of Health) and were normalized to $\beta$-actin. Final data were expressed as a percentage of the control.

\section{Data analysis}

All statistical procedures were performed on SPSS version 13.0 (SPSS Inc., Chicago, IL). All values were expressed as the mean \pm standard error of the mean (SEM). Data were evaluated by repeated-measures twoway analysis of variance (stress $\times$ treatment), while different treatment values were compared using $t$-tests where appropriate. Differences with a $P$ value less than 0.05 were considered statistically significant. 


\section{Results}

Effects of ginsenoside Rg1 in the sucrose consumption test

Sucrose consumption is an indicator of anhedonia-like behavioral change. The effects of ginsenoside $\operatorname{Rg} 1$ ( $40 \mathrm{mg} / \mathrm{kg}$ daily) pretreatment on sucrose preference test performance of CUMS-exposed rats are shown in Fig. 1. There were significant differences among the four groups regarding the percentage of sucrose consumption $\left(F_{(3,44)}=18.26, P<0.01\right)$. The percentage of sucrose consumption was significantly reduced in rats subjected to 5 -week CUMS exposure compared with the non-stressed control group, while chronic pretreatment with ginsenoside Rg1 (40 mg/kg daily) significantly increased the percentage of sucrose consumption in CUMS-exposed rats. Such results suggest a potent antidepressant-like effect of ginsenoside Rg1 in these CUMSexposed rats. No statistically significant differences were obtained between the vehicle-treated CUMS-exposed and CUMSexposed groups with regards to sucrose consumption $(P>0.05)$.

Effects of ginsenoside $\mathrm{Rg} 1$ in the forced swim test

Effects of ginsenoside Rg1 administrations on immobility times in the forced swim test are presented in Fig. 2. An overall significant difference was obtained among these groups with regards to immobility times $\left(F_{(3,44)}=17.38, P<\right.$ $0.01)$ and swimming times $\left(F_{(3,44)}=15.56\right.$, $P<0.01$ ) in this behavioral test. Results indicated that a 5-week CUMS exposure significantly increased immobility times while decreasing swimming times in

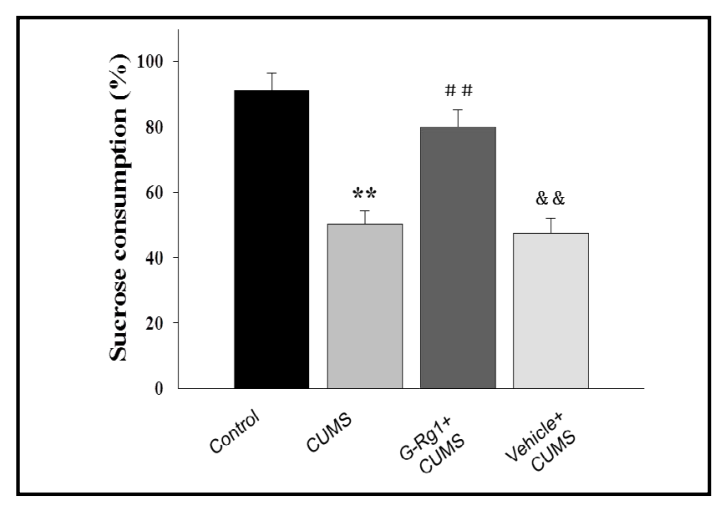

Fig. 1. Effects of ginsenoside $\operatorname{Rg} 1$ on the percentage of sucrose consumption. Ginsenoside Rg1 (40 mg/ $\mathrm{kg}$ ) alleviated decreased consumption of sucrose solution in CUMS-exposed rats in the sucrose preference test. All values are presented as the mean \pm SEM $(\mathrm{n}=12) .{ }^{* *} \mathrm{P}<0.01$ compared with the control

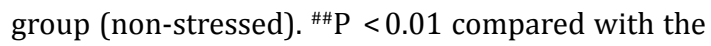
CUMS-exposed group. ${ }^{\&} \mathrm{P}<0.01$ compared with the control group (non-stressed). G-Rg1: ginsenoside Rg1.
Fig. 2. Effects of ginsenoside $\mathrm{Rg} 1$ on immobility times of rats in a forced swim test. Ginsenoside Rg1 (40 $\mathrm{mg} / \mathrm{kg}) \quad$ decreased immobility times (A) and increased swimming times (B), but exerted no statistically significant effects on struggling times (C) of CUMS-treated rats. All values are presented as the mean \pm SEM (n $=12$ ). ${ }^{* *} \mathrm{P}<0.01$ compared with the control group (non-stressed). \#\# $\mathrm{P}<0.01$ compared with the CUMS-exposed group. \&\& $\mathrm{P}<0.01$ compared with the control group (non-stressed). G-Rg1: ginsenoside Rg1.

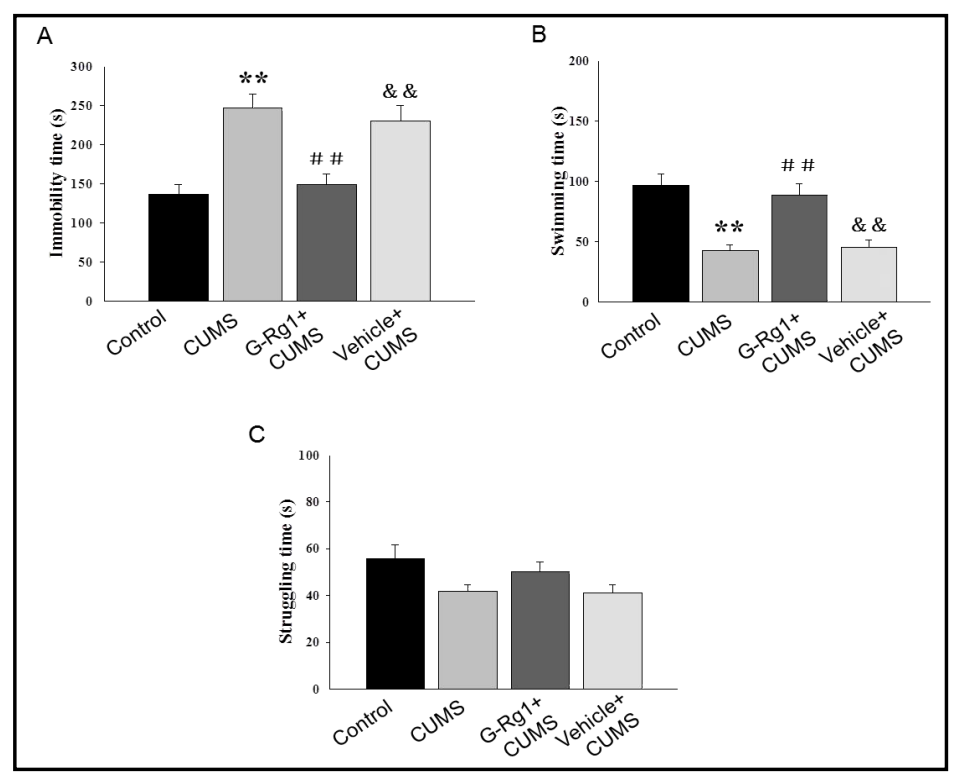




\section{Cellular Physiology Cell Physiol Biochem 2018;48:2470-2482 and Biochemistry \begin{tabular}{l|l} 
DOI: 10.1159/000492684 & $\begin{array}{l}\text { O 2018 The Author(s). Published by S. Karger AG, Basel } \\
\text { www.karger.com/cpb }\end{array}$
\end{tabular}

Fig. 3. Effects of ginsenoside $\operatorname{Rg} 1$ on locomotor activities in the open-field test. Each column represents the number of horizontal (white, crossings) or vertical exploratory (gray, rearing) activities measured in the 5 -min test period. All values are presented as the mean $\pm \operatorname{SEM}(\mathrm{n}=12) .{ }^{* *} \mathrm{P}<0.01$ compared with the control group (non-stressed). ${ }^{\# \#} \mathrm{P}<0.01$ compared with the CUMS-exposed group. \&\& $\mathrm{P}<0.01$ compared with the control group (nonstressed). G-Rg1: ginsenoside Rg1.

these rats compared with the nonstressed control group. In contrast, daily pretreatment with ginsenoside $\mathrm{Rg} 1$ in doses of $40 \mathrm{mg} / \mathrm{kg}$ significantly decreased immobility times and increased swimming times in comparison with the CUMSexposed animals. There were no significant differences between the vehicle-treated CUMS-exposed and CUMS-exposed groups with regards to immobility times $(P>$ 0.05 ). Additionally, there were no overall statistically significant differences among the four groups with regards to struggling behavior $(P>0.05)$. Such decreases in the duration of immobility suggest an antidepressant-like effect of ginsenoside Rg1 in these CUMS-exposed rats.

\section{Effects of ginsenoside Rg1 on locomotor activities in the open field test}

The effects of ginsenoside Rg1 administrations on horizontal locomotion (number of crossings) and vertical exploration (number of rearings) in the open-field test were recorded. The results, as summarized in Fig. 3, showed a statistically significant difference among the four groups in both the number of crossings $\left(F_{(3,44)}=14.85, \mathrm{p}<0.01\right)$ and rearings $\left(F_{(3,}\right.$ $\left.{ }_{44)}=12.91, \mathrm{p}<0.01\right)$. Five-week CUMS exposure significantly reduced the number of crossings and rearings compared with rats in the non-stressed control group. Whereas, chronic pretreatment with ginsenoside Rg1 ( $40 \mathrm{mg} / \mathrm{kg}$ daily) significantly increased the number of crossings and rearings in CUMSexposed rats compared with the CUMSexposed group. No statistically significant differences were obtained between the vehicle-treated CUMS-exposed group and
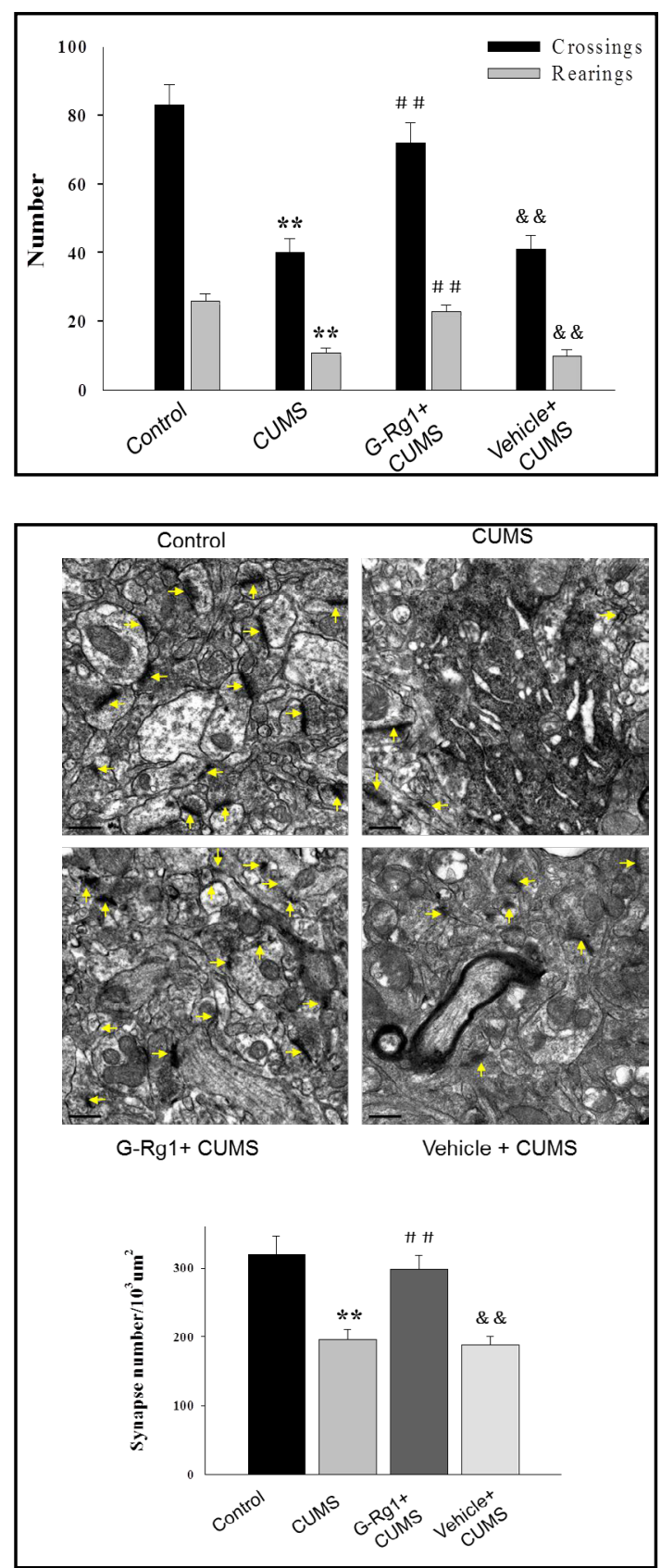

Fig. 4. Representative electron transmission micrographs of BLA neurons in rats from each group. Arrows indicate spine synapses. Scale bar, 0.5 $\mu \mathrm{m}$. Bar graph illustrating the mean synapse density of individual neurons within the BLA region. Error bars show SEM $(\mathrm{n}=4)$. ${ }^{* *} \mathrm{P}<0.01$ compared with the control group (non-stressed). "\# $\mathrm{P}<0.01$ compared with the CUMS-exposed group. ${ }^{\&} \mathrm{P}<0.01$ compared with the control group (non-stressed). G-Rg1: ginsenoside Rg1. 
CUMS-exposed control groups with regard to horizontal (crossings) and vertical (rearings) activities $(P>0.05)$. Again, these assays provided a third behavioral finding indicating that stress reduction occurred in the ginsenoside $\mathrm{Rg} 1$ pretreated rats.

\section{Effects of ginsenoside Rg1 on ultrastructure of the BLA}

The ultrastructure of neuronal synapses within the BLA was observed using transmission electron microscopy. Results revealed that there was an overall statistically significant difference in synaptic number density of BLA neurons among these four groups $\left(F_{(3,12)}=16.87, P<0.01\right)$. As shown in Fig. 4, the synaptic number density in the BLA of rats is significantly decreased after 5-week CUMS exposure compared with that of the non-stressed control group, while chronic pretreatment with ginsenoside Rg1 significantly ameliorated the decrease in synapses observed within the BLA of CUMS-exposed rats. No significant differences were obtained between the vehicle-treated CUMS-exposed and CUMS-exposed groups with regard to synapse numbers in the BLA $(P>0.05)$. In addition, mitochondrial degradation and vacuoles in the cytoplasm in CUMS-exposure rats were also ameliorated by ginsenoside $\operatorname{Rg} 1$ pretreatment. Such reductions in ultrastructural abnormalities demonstrate a potential neuroprotective effect of ginsenoside $\mathrm{Rg} 1$, which may involve one of the mechanisms that underlie its antidepressant-like effects.

\section{Effects of ginsenoside Rg1 on miR-134 expression in the BLA}

As miR-134 has the potential to modulate structural plasticity of synapses, we next examined miR-134 expression in the BLA region. Analysis revealed that there was a statistically significant difference among these groups with regards to the expression levels of miR-134 in BLA regions $\left(F_{(3,20)}=18.23, P<0.01\right)$. As shown in Fig. 5, expression of miR-

Fig. 5. Effects of ginsenoside $\operatorname{Rg} 1$ on miR-134 expressions in the BLA of rats. Band 1: control group; Band 2: CUMS group; Band 3: ginsenoside Rg1 (40 mg/kg) + CUMS group; Band 4: vehicle (0.9\% physiological saline, $10 \mathrm{ml} / \mathrm{kg})+$ CUMS group. Relative quantity of miR-134 versus U6 expressions in each group are presented as the mean \pm SEM ( $\mathrm{n}$ $=6)$. ${ }^{* *} \mathrm{P}<0.01$ compared with the control group (non-stressed). ${ }^{\#} \mathrm{P}<0.01$ compared with the CUMSexposed group. ${ }^{\&} \mathrm{P}<0.01$ compared with the control group (non-stressed). G-Rg1: ginsenoside Rg1.

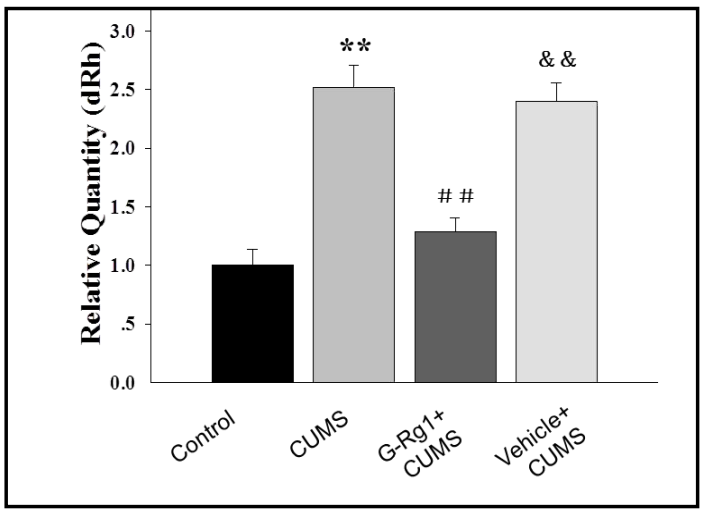

Fig. 6. Effects of ginsenoside $\operatorname{Rg} 1$ on the CREB protein expression and phosphorylation in the BLA of rats. Band 1: control group; Band 2: CUMS group; Band 3: ginsenoside Rg1 (40 mg/kg) + CUMS group; Band 4 : vehicle $0.9 \%$ physiological saline, $10 \mathrm{ml} / \mathrm{kg}$ ) + CUMS group. Phosphorylated-CREB levels were normalized to their corresponding total CREB levels and expressed as the mean \pm SEM $(\mathrm{n}=6)$. ** $\mathrm{P}<0.01$ compared with the control

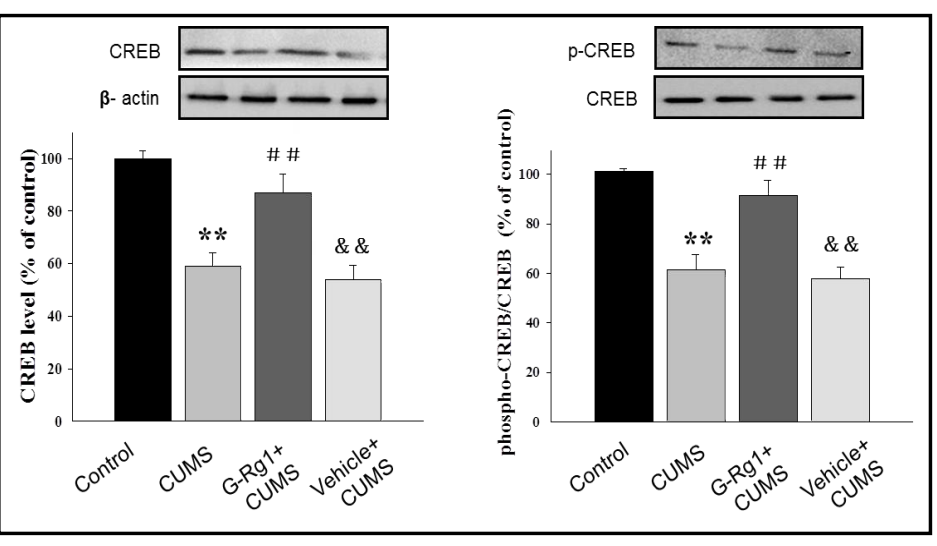
group (non-stressed). ${ }^{\# \# P}<0.01$ compared with the CUMS-exposed group. ${ }^{\& \&}<<0.01$ compared with the control group (non-stressed). G-Rg1: ginsenoside $\operatorname{Rg} 1$.

\section{KARGER}




\section{Cellular Physiology Cell Physiol Biochem 2018;48:2470-2482

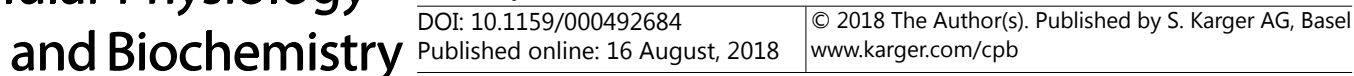

134 in the BLA was significantly reduced after 5 weeks of CUMS exposure compared with that of the non-stressed control group, while chronic pretreatment of ginsenoside Rg1 significantly increased the expression of miR-134 in the BLA of CUMS-exposed rats. No significant differences were obtained between the vehicle-treated CUMS-exposed group and CUMS-exposed groups with regards to the expression of miR-134 in the BLA $(P>0.05)$. These results indicate that the miR-134 signaling pathway within the BLA may be involved in the antidepressantlike effects of ginsenoside $\operatorname{Rg} 1$ in these depressed rats.

\section{Effects of ginsenoside Rg1 on CREB} activity levels in the BLA

To determine whether ginsenoside Rg1 treatment affected synaptic-associated proteins, expression and phosphorylation levels of CREB, a nuclear transcription factor that has been reported to be a key mediator of cell survival, were assessed in the BLA of these rats. Western blot analysis showed that there was an overall significant difference in phosphorylation levels of CREB within the BLA regions among the four groups $\left(F_{(3,20)}=17.09, P<0.01\right)$. As shown in Fig. 6, there was a significant decrease in the

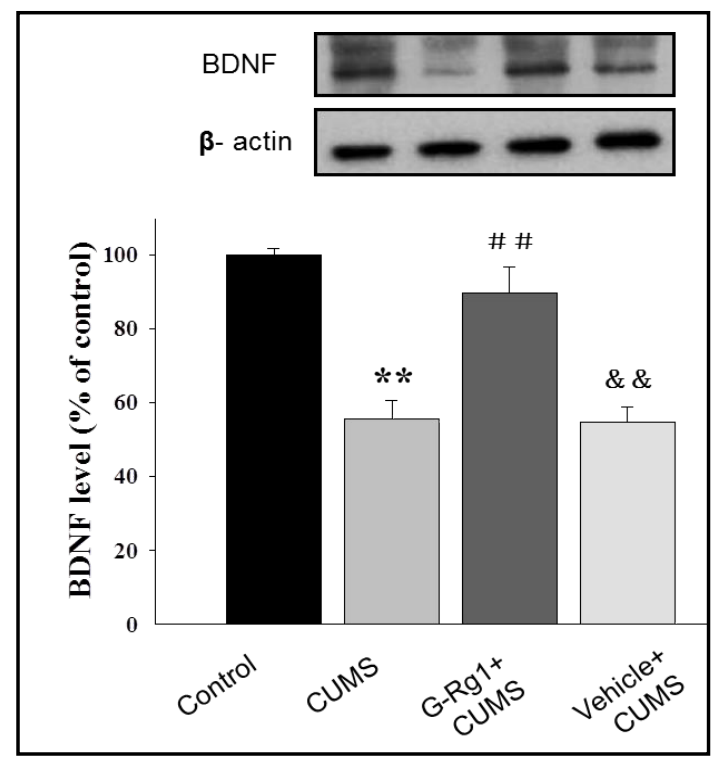

Fig. 7. Effects of ginsenoside $\mathrm{Rg} 1$ on BDNF protein expression in the BLA of rats. Band 1: control group; Band 2: CUMS group; Band 3: ginsenoside Rg1 $(40 \mathrm{mg} / \mathrm{kg})+$ CUMS group; Band 4: vehicle $(0.9 \%$ physiological saline, $10 \mathrm{ml} / \mathrm{kg}$ ) + CUMS group. Normalized intensity bands of BDNF were presented as the mean \pm SEM $(n=12) .{ }^{* *} \mathrm{P}<0.01$ compared with the control group (non-stressed). ${ }^{\#} P<0.01$ compared with the CUMS-exposed group. ${ }^{\text {\&\&}} \mathrm{P}<0.01$ compared with the control group (non-stressed). G-Rg1: ginsenoside Rg1. expression and phosphorylation of CREB protein in the CUMS-exposed rats compared with that of the non-stressed control group. These decreases were significantly ameliorated in rats pretreated with ginsenoside Rg1. No significant differences were observed between the vehicle-treated CUMS-exposed and CUMS-exposed groups with regards to CREB protein expression and phosphorylation levels in the BLA $(P>0.05)$. Such reductions in BLA CREB protein expression and phosphorylation of CUMS-exposed rats by ginsenoside Rg1 reveal an additional potentially important mechanism involved in the antidepressant-like effects of this agent.

\section{Effects of ginsenoside Rg1 on BDNF protein levels in the BLA}

To further determine whether neurotrophic factors are involved in the anti-depressant effects induced by ginsenoside Rg1, protein expression levels of BDNF in the BLA were investigated. Statistical analysis of BDNF expression within the BLA also showed an overall significant difference among these groups $\left(F_{(3,20)}=17.28, P<0.01\right)$. As shown in Fig. 7 , western blot assays demonstrate that exposure to a 5-week-period of CUMS resulted in a significant decrease in BLA BDNF protein levels in comparison with that observed in the non-stressed control group, while chronic pretreatment with ginsenoside Rg1 significantly ameliorated this CUMS-induced downregulation of BDNF. No significant differences were obtained between the vehicle-treated CUMS-exposed and CUMS-exposed control groups with regards to the BDNF protein levels in the BLA $(P>0.05)$. These results indicate that ginsenoside Rg1 can upregulate BDNF levels within the BLA of CUMS-exposure rats, suggesting another possible mechanism through which ginsenoside Rg1 may exert antidepressant-like effects. 


\section{Cellular Physiology Cell Physiol Biochem 2018;48:2470-2482 \\ and Biochemistry Published online: 16 August, 2018 \begin{tabular}{l|l} 
DOI: 10.1159/000492684 2018 The Author(s). Published by S. Karger AG, Basel \\
www.karger.com/cpb
\end{tabular}}

Yu et al.: Ginsenoside Rg1 Prevents Depression Phenotypes

\section{Discussion}

Despite an increasing number of studies demonstrating a relationship between depression and structural plasticity in specific brain regions, no effective therapeutic tools with antidepressant-like properties have yet been developed. In this study, we evaluated the effects of chronic stress and pretreatment with the traditional herbal agent ginsenoside Rg1 on the expression of a variety of key regulators of structural synaptic plasticity and depression-like behaviors in rats. Results from our behavioral assays, including sucrose preference and forced swim tests, showed that CUMS induces depression-like behaviors in rats, consistent with the findings presented in previous reports [40, 41]. These behavioral responses, which are indicators of depression, were accompanied by significant structural changes in dendritic spines and synapses within the BLA. More importantly, chronic treatment with ginsenoside Rg1 significantly ameliorated these behavioral and neuronal structural changes, while markedly reducing expression levels of miR-134, CREB, and BDNF in the BLA of these CUMS rats. These findings suggested that ginsenoside $\operatorname{Rg} 1$ may exhibit neuroprotective and antidepressant-like effects via the activation of signaling pathways associated with synaptic plasticity in this CUMS-induced depression model.

The CUMS-induced animal model of depression is widely used in behavioral assays such as the sucrose preference and forced swim tests for evaluating the efficacy of chronic antidepressant treatments $[42,43]$. The sucrose preference test serves as an index of anhedonia-like behavior. Specifically, reduction in sucrose solution consumption is associated with depression, while the restoration of this response is observed in response to chronic antidepressant treatments. This study showed that rats subjected to 5 weeks of CUMS exposure consumed decreased amounts of sucrose solution in comparison with the non-stressed group, while chronic pretreatment with ginsenoside Rg1 significantly prevented this behavioral change. The forced swim test is used for assessing "behavioral despair", another core symptom of major depressive disorders. In this test, an increase in immobility time is interpreted as an indicator of depression. Our results showed that 5 weeks of CUMS exposure caused significant increases in immobility times in the forced swim test. Ginsenoside $\operatorname{Rg} 1$ pretreatment dramatically decreased immobility times while significantly increasing swimming times of CUMS-exposed rats, which were consistent with results from previous studies $[44,45]$. Similarly, in the open-field test, CUMS exposure significantly reduced the number of crossings and rearings, suggesting a loss of exploration and interest to a novel environment [46]. This reduction in locomotor activity was also ameliorated by chronic pretreatment of ginsenoside $\mathrm{Rg} 1$. Taken together, these behavioral results suggested that ginsenoside Rg1 treatment exerts antidepressant-like effects in this CUMS-induced animal model of depression.

This study also showed that antidepressant-like effects of ginsenoside Rg1 in this CUMS-induced depression model were accompanied by a dramatic amelioration of neuronal structural changes in the BLA, a critical structure considered to be associated with depression. It has recently been demonstrated that depression is associated with a range of neuronal structural changes in several brain regions [47, 48]. For example, chronic stress induces morphological changes in dendrites and spines in hippocampal pyramidal $[49,50]$ and prefrontal cortex neurons [51,52], as well as causing changes in synaptic parameters within the amygdala [53], all of which are associated with structural plasticity changes in depression. In the present study, we focused on the BLA, another important region associated with depression. At this site, we investigated the relationship between neuronal structural plasticity and antidepressant-like effects of ginsenoside Rg1. The results of our ultrastructural analysis using electron microscopy showed that 5 weeks of CUMS exposure lead to significant ultrastructural changes in BLA neurons consisting of decreases in synaptic number, surface density and thickness of post synaptic density. Such alterations indicate some of the ultrastructural abnormalities in BLA that may be involved in the underlying mechanisms of depression-like behaviors induced by chronic stress. More importantly, long-term ginsenoside Rg1 treatment ameliorated these ultrastructural abnormalities 


\section{Cellular Physiology Cell Physiol Biochem 2018;48:2470-2482 \\ \begin{tabular}{l|l|l} 
DOI: 10.1159/000492684 & $\begin{array}{l}\text { O 2018 The Author(s). Published by S. Karger AG, Basel } \\
\text { www.karger.com/cpb }\end{array}$
\end{tabular}}

Yu et al.: Ginsenoside Rg1 Prevents Depression Phenotypes

in BLA neurons, thus preventing depression-like behaviors in these CUMS-exposed rats. Collectively, these results provide evidence suggesting that the potential antidepressant-like mechanisms of ginsenoside Rg1 may involve its neuroprotective effects related to synaptic structural plasticity.

To further explore the signaling pathways underlying potential neuroprotective events associated with the antidepressant-like effects of ginsenoside Rg1, we examined miR-134 expression. miR-134 negatively regulates synaptic plasticity by inhibiting translation of key plasticity proteins. Specifically, the presence of miR-134 within dendrites at post-synaptic sites suggests a possible functional role that may be involved in the regulation of synapse structure and function [15]. Our results reveal that the antidepressant-like effects of ginsenoside $\mathrm{Rg} 1$ in this CUMS model of depression were accompanied by changes in miR-134 expression within the BLA. Specifically, we found that 5 weeks of CUMS exposure significantly increased miR-134 levels in the BLA. Long-term pretreatment with ginsenoside Rg1 significantly attenuated miR-134 overexpression, which was accompanied by amelioration of depressionlike behaviors in these CUMS-exposed rats. These results suggest that miR-134 may act as a negative regulator of dendritic synapse morphology in BLA neurons subjected to chronic stress, and in this way, reveals an additional neuroprotective mechanism of ginsenoside Rg1 in producing antidepressant-like effects.

We also found that increases in miR-134 expression in response to CUMS exposure were accompanied by significant down-regulation of CREB and BDNF in the BLA. Again, systemic administration of ginsenoside $\mathrm{Rg} 1$ effectively prevented the deficits of these proteins induced by CUMS in the BLA. CREB is considered to be one of the key potential target molecules activated downstream of miR-134, and activation of CREB will in turn lead to increased BDNF gene expression [54, 55]. Previous studies have reported that ginsenoside Rg1 could upregulate the BDNF signaling pathway within the hippocampus, preventing decreases in dendritic spine density resulting from chronic stress in mice [32]. These findings suggest that a distinct signaling pathway downstream of miR-134 may account for the ginsenoside Rg1mediated neurotrophic effects in these CUMS-exposed rats. Our current results demonstrate that ginsenoside Rg1 increased CREB phosphorylation and BDNF expression accompanied by decreased expression of miR-134. When combining these findings with those from our ultrastructure assays, our results indicate that the miR-134-CREB-BDNF signal transduction cascade may contribute to the structural and/or functional regulation of neurons in the BLA by ginsenoside Rg1. Thus, these data strongly support the hypothesis that CREB-BDNF cascade activation may act as a positive candidate for the miR-134 regulated neuroprotective effects of ginsenoside $\operatorname{Rg} 1$ upon amelioration of CUMS-induced depression-like behaviors. In this way, ginsenoside Rg1 may suppress miR-134 activation, thereby up-regulating the expression of its downstream signaling components, the synaptic plasticity-related CREB and BDNF proteins within BLA neurons, to produce the morphological changes of neuronal synapses that can then ameliorate depression-like behaviors in rats.

\section{Conclusion}

The results of this study suggest that chronic administration of ginsenoside $\operatorname{Rg} 1$ is effective in ameliorating depression-like behaviors, which may in part involve distinct neuroprotective effects in this CUMS-treated animal model of depression. Specifically, this study provides the first in vivo finding that one of the possible mechanisms for the antidepressant effects of ginsenoside $\operatorname{Rg} 1$ involves amelioration of dysregulation in neuronal structural plasticity within the BLA. These findings provide a novel potential regimen by which herbal antidepressants may exert therapeutic effects in the treatment of depression. 


\section{Cellular Physiology Cell Physiol Biochem 2018;48:2470-2482 \begin{tabular}{ll|l} 
and Biochemistry Published online: 16 August, 2018 & $\begin{array}{l}\text { (c) } 2018 \text { The Author(s). Published by S. Karger AG, Basel } \\
\text { www.karger.com/cpb }\end{array}$
\end{tabular}}

Yu et al.: Ginsenoside Rg1 Prevents Depression Phenotypes

\section{Acknowledgements}

This study was supported by a grant to Xueqin Mao from the Natural Science Foundation of Shandong Province (ZR2013HM092) and grants to Shu Yan Yu from the National Natural Science Foundation of China (NSFC81471371) and the Key Research and Development Foundation of Shandong Provincial (2015GGH318024).

\section{Disclosure Statement}

The authors declare that they have no actual or potential conflicts of interest associated with the conduct of this work.

\section{References}

1 Vyas A, Mitra R, Shankaranarayana Rao BS, Chattarji S: Chronic stress induces contrasting patterns of dendritic remodeling in hippocampal and amygdaloid neurons. J Neurosci 2002;22:6810-6818.

2 Stockmeier CA, Mahajan GL, Konick LC, Overholser JC, Jurjus GJ, Meltzer HY, Uylings HB, Friedman L, Rajkowska G: Cellular changes in the postmortem hippocampus in major depression. Biol Psychiatry 2004;56:640-650.

3 Oh DH, Son H, Hwang S, Kim SH: Neuropathological abnormalities of astrocytes, GABAergic neurons, and pyramidal neurons in the dorsolateral prefrontal cortices of patients with major depressive disorder. Eur Neuropsychopharmacol 2012;22:330-338.

4 Davidson RJ, Irwin W, Anderle MJ, Kalin NH: The neural substrates of affective processing in depressed patients treated with venlafaxine. Am J Psychiatry 2003;160:64-75.

-5 Sheline YI, Barch DM, Donnelly JM, Ollinger JM, Snyder AZ, Mintun MA: Increased amygdala response to masked emotional faces in depressed subjects resolves with antidepressant treatment: an fMRI study. Biol Psychiatry 2001;50:651-658.

6 Bogdan R, Williamson DE, Hariri AR: Mineralocorticoid receptor Iso/Val (rs5522) genotype moderates the association between previous childhood emotional neglect and amygdala reactivity. Am J Psychiatry 2012;169:515-522.

7 Dannlowski U, Stuhrmann A, Beutelmann V, Zwanzger P, Lenzen T, Grotegerd D, Domschke K, Hohoff C, Ohrmann P, Bauer J, Lindner C, Postert C, Konrad C, Arolt V, Heindel W, Suslow T, Kugel H: Limbic scars: long-term consequences of childhood maltreatment revealed by functional and structural magnetic resonance imaging. Biol Psychiatry 2012;71:286-293.

-8 Armony JL, Corbo V, Clément MH, Brunet A: Amygdala response in patients with acute PTSD to masked and unmasked emotional facial expressions. Am J Psychiatry 2005;162:1961-1963.

-9 van Wingen GA, Geuze E, Vermetten E, Fernández G: Perceived threat predicts the neural sequelae of combat stress. Mol Psychiatry 2011;16:664-671.

-10 Adamec R, Hebert M, Blundell J, Mervis RF: Dendritic morphology of amygdala and hippocampal neurons in more and less predator stress responsive rats and more and less spontaneously anxious handled controls. Behav Brain Res 2012;226:133-146.

11 Hill MN, Hillard CJ, McEwen BS: Alterations in corticolimbic dendritic morphology and emotional behavior in cannabinoid CB1 receptor-deficient mice parallel the effects of chronic stress. Cereb Cortex 2011;21:2056-2064.

12 Vyas A, Mitra R, Shankaranarayana Rao BS, Chattarji S: Chronic stress induces contrasting patterns of dendritic remodeling in hippocampal and amygdaloid neurons. J Neurosci 2002;22:6810-6818.

13 Bredy TW, Lin Q, Wei W, Baker-Andresen D, Mattick JS: MicroRNA regulation of neural plasticity and memory. Neurobiol Learn Mem 2011;96:89-94.

14 Presutti C, Rosati J, Vincenti S, Nasi S: Non coding RNA and brain. BMC Neurosci 2006;7:S5.

15 Schratt GM, Tuebing F, Nigh EA, Kane CG, Sabatini ME, Kiebler M, Greenberg, ME: A brain-specific microRNA regulates dendritic spinedevelopment. Nature 2006;439:283-289. 


\section{Cellular Physiology Cell Physiol Biochem 2018;48:2470-2482 and Biochemistry \begin{tabular}{l|l} 
DOI: 10.1159/000492684 & (c) 2018 The Author(s). Published by S. Karger AG, Basel \\
www.karger.com/cpb
\end{tabular}

16 Gao J, Wang WY, Mao YW, Graff J, Guan JS, Pan L, Mak G, Kim D, Su SC, Tsai LH: A novel pathway regulates memory and plasticity via SIRT1 and miR-134. Nature 2010;466:1105-1109.

17 Silva AJ, Kogan JH, Frankland PW, Kida S: CREB and memory. Annu Rev Neurosci 1998;21:127-148.

18 Li P, Rial D, Canas PM, Yoo JH, Li W, Zhou X, Wang Y, van Westen GJ, Payen MP, Augusto E, Gonçalves N, Tomé AR, Li Z, Wu Z, Hou X, Zhou Y, IJzerman AP, Boyden ES, Cunha RA, Qu J, et al.: Optogenetic activation of intracellular adenosine A2A receptor signaling in the hippocampus is sufficient to trigger CREB phosphorylation and impair memory. Mol Psychiatry 2015;20:1339-1349.

19 Huang EJ, Reichardt LF: Neurotrophins: roles in neuronal development and function. Annu Rev Neurosci 2001;24:677-736.

20 Leal G, Comprido D, Duarte CB: BDNF-induced local protein synthesis and synaptic plasticity. Neuropharmacology 2014;76:639-656.

21 Wei CJ, Augusto E, Gomes CA, Singer P, Wang Y, Boison D, Cunha RA, Yee BK, Chen JF: Regulation of fear responses by striatal and extrastriatal adenosine A2A receptors in forebrain. Biol Psychiatry. 2014;75:855863.

-22 Nibuya M, Morinobu S, Duman RS: Regulation of BDNF and trkB mRNA in rat brain by chronic electroconvulsive seizure and antidepressant drug treatments. J Neurosci 1995;15:7539-7547.

-23 Karege F, Perret H, Bondolfi G, Schwald M, Bertschy G, Aubrey JM: Decreased serum brain-derived neurotrophic factor levels in major depressed patients. Psychiatry Res 2002;109:143-148.

-24 Guilloux JP, Douillard-Guilloux G, Kota R, Wang X, Gardier AM, Martinowich K, Tseng GC, Lewis DA, Sibille E: Molecular evidence for BDNF- and GABA-related dysfunctions in the amygdala of female subjects with major depression. Mol Psychiatry 2012;17:1130-1142.

25 Berton 0, Nestler EJ: New approaches to antidepressant drug discovery: Beyond monoamines. Nat Rev Neurosci 2006;7:137-151.

-26 Nemeroff CB: The burden of severe depression: a review of diagnostic challenges and treatment alternatives. J Psychiatr Res 2007;41:189-206.

-27 Van der Watt G, Laugharne J, Janca A: Complementary and alternative medicine in the treatment of anxiety and depression. Curr Opin Psychiatry 2008;21:37-42.

28 Zhang X, Wang J, Xing Y, Gong L, Li H, Wu Z, Li Y, Wang J, Wang Y, Dong L, Li S: Effects of ginsenoside Rg1 or17 $\beta$-estradiol on a cognitively impaired, ovariectomized rat model of Alzheimer's disease. Neuroscience 2012;220:191-200.

29 Song XY, Hu JF, Chu SF, Zhang Z, Xu S, Yuan YH, Han N, Liu Y, Niu F, He X, Chen NH: Ginsenoside Rg1 attenuates okadaic acid induced spatial memory impairment by the GSK3 $\beta /$ tau signaling pathway and the $A \beta$ formation prevention in rats. Eur J Pharmacol 2013;710:29-38.

30 Wang Y, Kan H, Yin Y, Wu W, Hu W, Wang M, Li W, Li W: Protective effects of ginsenoside Rg1 on chronic restraint stress induced learning and memory impairments in male mice. Pharmacol Biochem Behav 2014;120:73-81.

31 Zhu J, Mu X, Zeng J, Xu C, Liu J, Zhang M, Li C, Chen J, Li T, Wang Y: Ginsenoside Rg1 prevents cognitive impairment and hippocampus senescence in a rat model of D-galactose-induced aging. PLoS One 2014;9:e101291.

-32 Jiang B, Xiong Z, Yang J, Wang W, Wang Y, Hu ZL, Wang F, Chen JG: Antidepressant-like effects of ginsenoside Rg1 are due to activation of the BDNF signalling pathway and neurogenesis in the hippocampus. $\mathrm{Br} \mathrm{J}$ Pharmacol 2012;166:1872-1887.

-33 Xie CL, Li JH, Wang WW, Zheng GQ, Wang LX: Neuroprotective effect of ginsenoside-Rg1 on cerebral ischemia/reperfusion injury in rats by downregulating protease-activated receptor-1 expression. Life Sci 2015;121:145-151.

34 Mao QQ, Ip SP, Ko KM, Tsai SH, Che CT: Peony glycosides produce antidepressant-like action in mice exposed to chronic unpredictable mild stress: effects on hypothalamic-pituitary-adrenal function and brain-derived neurotrophic factor. Prog Neuropsychopharmacol Biol Psychiatry 2009;33:1211-1216.

35 Porsolt RD, Le Pichon M, Jalfre M: Depression: a new animal model sensitive to antidepressant treatments. Nature 1977;266:730-732.

-36 Duman CH, Schlesinger L, Kodama M, Russell DS, Duman RS: A role for MAP kinase signaling in behavioral models of depression and antidepressant treatment. Biol Psychiatry 2007;61:661-670.

-37 Walsh RN, Cummins RA: The open-field test: a critical review. Psychol Bull 1976;83:482-504. 


\section{Cellular Physiology Cell Physiol Biochem 2018;48:2470-2482 \begin{tabular}{ll|l} 
DOI: 10.1159/000492684 & $\begin{array}{l}\text { O 2018 The Author(s). Published by S. Karger AG, Basel } \\
\text { www.karger.com/cpb }\end{array}$
\end{tabular}}

Yu et al.: Ginsenoside Rg1 Prevents Depression Phenotypes

-38 DeFelipe J, Marco P, Busturia I, Merchán-Pérez A: Estimation of the number of synapses in the cerebral cortex: methodological considerations. Cereb Cortex 1999;9:722-732.

-39 Merchán-Pérez A, Rodriguez JR, Alonso-Nanclares L, Schertel A, Defelipe J: Counting Synapses Using FIB/SEM Microscopy: A True Revolution for Ultrastructural Volume Reconstruction. Front Neuroanat 2009;3:18.

40 Willner P: Chronic mild stress (CMS) revisited: consistency and behavioural-neurobiological concordance in the effects of CMS. Neuropsychobiology 2005;52:90-110.

-41 Zhao Z, Wang W, Guo H, Zhou D: Antidepressant-like effect of liquiritin from Glycyrrhiza uralensis in chronic variable stress induced depression model rats. Behav Brain Res 2008;194:108-113.

$>42$ Nestler EJ, Hyman SE: Animal models of neuropsychiatric disorders. Nat Neurosci 2010;13:1161-1169.

-43 Christiansen SH, Olesen MV, Wörtwein G, Woldbye DP: Fluoxetine reverts chronic restraint stress-induced depression-like behaviour and increases neuropeptide $Y$ and galanin expression in mice. Behav Brain Res 2011;216:585-591.

44 Zhu X, Gao R, Liu Z, Cheng Z, Qi Y, Fan C, Yu SY: Ginsenoside Rg1 reverses stress-induced depression-like behaviors and BDNF expression within the prefrontal cortex. Eur J Neurosci 2016;44:1878-1885.

45 Liu Z, Qi Y, Cheng Z, Zhu X, Fan C, Yu SY: The effects of ginsenoside Rg1 on chronic stress induced depression-like behaviors, BDNF expression and the phosphorylation of PKA and CREB in rats. Neuroscience 2016;322:358-369.

46 Katz RJ, Roth KA, Carroll BJ: Acute and chronic stress effects on open field activity in the rat: implications for a model of depression. Neurosci Biobehav Rev 1981;5:247-251.

47 Christoffel DJ, Golden SA, Russo SJ: Structural and synaptic plasticity in stress-related disorders. Rev Neurosci 2011;22:535-549.

48 Popoli M, Yan Z, McEwen BS, Sanacora G: The stressed synapse: the impact of stress and glucocorticoids on glutamate transmission. Nat Rev Neurosci 2011;13:22-37.

49 McEwen BS: The neurobiology of stress: from serendipity to clinical relevance. Brain Res 2000;886:172189.

50 Donohue HS, Gabbott PL, Davies HA, Rodríguez JJ, Cordero MI, Sandi C, Medvedev NI, Popov VI, Colyer FM, Peddie CJ, Stewart MG: Chronic restraint stress induces changes in synapse morphology in stratum lacunosum-moleculare CA1 rat hippocampus: a stereological and three-dimensional ultrastructural study. Neuroscience 2006;140:597-606.

51 Goldwater DS, Pavlides C, Hunter RG, Bloss EB, Hof PR, McEwen BS, Morrison JH: Structural and functional alterations to rat medial prefrontal cortex following chronic restraint stress and recovery. Neuroscience 2009;164:798-808.

52 Cook SC, Wellman CL: Chronic stress alters dendritic morphology in rat medial prefrontal cortex. J Neurobiol 2004;60:236-248.

53 Zhang L, Luo JX, Zhang M, Yao W, Ma X, Yu SY: Effects of curcumin on chronic unpredictable mild stress-induced depressive-like behavior and structural plasticity in the lateral amygdala of rats. Int J Neuropsychopharmacol 2014;17:793-806.

54 Yamada K, Nabeshima T: Brain-derived neurotrophic factor/TrkB signaling in memory processes. J Pharmacol Sci 2003;91:267-270.

55 Tao X, Finkbeiner S, Arnold DB, Shaywitz AJ, Greenberg ME: Calcium influx regulates BDNF transcription by a CREB family transcription factor-dependent mechanism. Neuron 1998;20:709-726. 